\title{
Arthrobotrys robusta no controle biológico de nematóides parasitos gastrintestinais de bovinos
}

\section{Arthrobotrys robusta in biological control of bovine nematode gastrointestinal parasites}

\author{
Alessandra Pereira Simonini Gomes, Jackson Victor de Araújo*
}

\begin{abstract}
Resumo
Um estudo foi realizado com o objetivo de testar o potencial in vivo do fungo predador de nematóides Arthrobotrys robusta em predar e controlar nematóides parasitos gastrintestinais de bovinos a pasto. Vinte bezerros foram tratados previamente com Albendazole e divididos em dois piquetes naturalmente infestados por helmintos gastrintestinais, um grupo controle (grupo A) e um grupo tratado com conídios (grupo B). Uma semana depois, cada bezerro recebeu por via oral 10 mil larvas infectantes de Haemonchus placei. No grupo B, cada animal recebeu 2 milhões de conídios de $A$. robusta, por via oral, duas vezes por semana, durante quatro meses, a partir do início da estação chuvosa da região (outubro). Os animais do grupo A não receberam conídios. Amostras de fezes e de pasto foram coletadas a cada 14 dias durante todo o período experimental. Dados de temperatura, umidade relativa do ar e precipitação pluvial foram coletados. Um bezerro traçador foi introduzido a cada 15 dias em cada piquete. Houve uma redução de $73,68 \%$ no número de ovos por grama de fezes nos bovinos do grupo $B$, quando comparados aos do grupo $A$ em maio $(P<0,05)$. Cooperia foi o gênero mais prevalente em ambos os grupos, no número de larvas recuperadas das necrópsias dos animais traçadores e nas pastagens, seguindo os gêneros Haemonchus e Oesophagostomum. Houve uma redução de $77,39 \%$ na contagem de larvas recuperadas da pastagem do grupo $B(P<0,05)$, quando comparado ao grupo $A$.
\end{abstract}

Palavras-chave: controle biológico; fungos nematófagos; fungos predadores de nematóides; Arthrobotrys robusta, nematóides; bovinos.

\begin{abstract}
A study was carried out to investigate the in vivo potential of the nematode-trapping fungi Arthrobotrys robusta, in trapping and controlling, at pasture soil, bovine gastrointestinal parasitic nematodes. Twenty calves were treated previously with albendazol and later divided into two pastures naturally infested by gastrointestinal helminth, one for the control group (group A) and one for the conidia treated group (group B). One week after each calf received by oral route 10,000 infective Haemonchus placei larvae. In group $\mathrm{B}$, each animal received two million conidia of $A$. robusta, orally, twice a week, during four months period that started together with the region's rainy season (october). The animals in group A did not receive any conidia. Fecal and pasture soil samples were collected for parasitic examination every 14 days, during all the experimental period. Temperature, relative air humidity and rainfall data were recorded. One tracer calf was introduced every 15 days in each pasture. The results showed $73,68 \%$ reduction in the number of eggs per gram of faeces in the bovine of the group $B$, when compared to group $A$, in May $(P<0.05)$. Cooperia was the most prevalent genus in both groups, in the number of larvae recovered from necropsy of tracer animals and in pasture, followed by Haemonchus and Oesophagostomum genera. There was a reduction of $77,39 \%$ in larvae counts recovered from pasture in group $B(P<0.05)$, when compared to group $A$.
\end{abstract}

Keywords: biological control; nematophagous fungi; nematode-trapping fungi; Arthrobotrys robusta; nematodes; bovine.

\section{Introdução}

O parasitismo gastrintestinal por helmintos é um dos mais importantes problemas na produtividade de ruminantes em todo o mundo. Sua presença causa uma condição subclínica onde os efeitos mais evidentes estão nos animais jovens, os quais ainda não adquiriram resistência imunológica a esses agentes.
Os vários estádios do parasito no meio ambiente representam infecções futuras e apresentam estratégias de sobrevivência e comportamento, cuja finalidade é o encontro com um novo hospedeiro. Os esforços de controle devem ser concentrados no ambiente, interrompendo o processo pelo qual a infecção potencial torna-se uma infecção no animal. O controle biológico visa a reduzir os estádios de vida livre dos nematóides parasitos de animais domésticos nas pastagens.

Departamento de Veterinária, Universidade Federal de Viçosa, CEP 36571-000, Viçosa-MG, Brazil, Phone: 550318991464 ,

Fax: 550318992314 , e-mail: jvictor@mail.ufv.br

* Autor de correspondência 
Os fungos nematófagos predadores formam armadilhas ao longo da hifa que capturam os nematóides. As redes tridimensionais são as mais comuns das estruturas de captura, sendo encontradas em várias espécies do gênero Arthrobotrys. Após a captura, independentemente da armadilha utilizada, o fungo penetra e se desenvolve no interior do nematóide, consumindo o seu conteúdo e lançando para o meio externo as suas estruturas vegetativas e reprodutivas (Barron, 1977 ; Gray, 1987).

Espera-se que o controle biológico de nematóides por fungos possa ser empregado em períodos de maior disponibilidade de larvas infectantes na pastagem, reduzindo a ingestão pelos animais e, conseqüentemente, a incidência de casos clínicos, além de promover a imunidade dos animais através da ingestão de pequeno número de larvas.

O objetivo deste trabalho foi o de utilizar um isolado de Arthrobotrys robusta no controle biológico de nematóides parasitos gastrintestinais de bovinos infectados experimentalmente com Haemonchus placei e naturalmente por nematóides presentes no pasto.

\section{Material e métodos}

Larvas infectantes $\left(L_{3}\right)$ de Haemonchus placei foram gentilmente cedidas pelo Departamento de Parasitologia da Universidade de São Paulo.

Panagrellus (nematóides de vida livre) foram mantidos em placas de Petri com meio de aveia em flocos, umedecida e amassada. Este nematóide foi extraído do meio de cultura através da imersão de pequenas quantidades de aveia em água destilada no funil de Baermann e coletado em tubos de hemólise após seis horas de decantação.

Para obtenção de nematóides sem bactérias fecais e fungos, estes foram lavados com água destilada e centrifugados a $1.000 \mathrm{rpm}$, por cinco minutos, desprezando no final de cada centrifugação o sobrenadante. Posteriormente, os $H$. placei foram mantidos à temperatura ambiente, por uma semana, em solução contendo $0,05 \%$ de sulfato de estreptomicina, $0,05 \%$ de cloranfenicol e $0,05 \%$ de anfotericina $B$, sendo repetido o processo de lavagem em água destilada, como descrito anteriormente (Araújo, 1996).

Um isolado de fungo predador da espécie Arthrobotrys robusta, oriundo de solo do Brasil, foi obtido pelo método do espalhamento do solo de Duddington (1955) e modificado por Santos et al. (1991). Este isolado foi repicado mensalmente e conservado em tubos de ensaio contendo batatadextrose-ágar $2 \%$, a $4^{\circ} \mathrm{C}$ e no escuro. Para induzir a formação de conídios de $A$. robusta, sua produção foi realizada em meio ágar-fezes segundo técnica descrita por Araújo (1996).

O experimento foi conduzido em propriedade da Universidade Federal de Viçosa, no Município de Viçosa, Estado de Minas Gerais, Região Sudeste do Brasil, durante o período de outubro de 1996 a maio de 1997.

Vinte bezerros machos, mestiços holandês $x$ zebu, de seis meses de idade, foram colocados em pastagens de capimgordura (Melinis minutiflora) naturalmente infestadas por helmintos parasitos gastrintestinais. Estes animais foram tratados, previamente, com duas doses de $7,5 \mathrm{mg} / \mathrm{kg}$ de peso vivo de albendazole, intervaladas de duas semanas, sendo introduzidos na pastagem uma semana após o término do tratamento. Posteriormente, cada um dos bezerros recebeu, por via oral, $10.000 \mathrm{~L}_{3}$ de $H$. placei.

Os animais foram divididos, aleatoriamente, em dois pastos contendo dez bezerros em cada um deles, com taxa de lotação de 1,7 animal/hectare, formando, então, os grupos controle $(A)$ e tratado (B). No grupo $A$, os bezerros não receberam conídios de fungos. No grupo $B$, cada animal recebeu $2 \times 10^{6}$ conídios de $A$. robusta, por via oral, duas vezes por semana, por um período de quatro meses a partir do começo do período chuvoso na região (outubro). De catorze em catorze dias, após a administração das $L_{3}$ aos bezerros, nos animais de cada grupo foram colhidas amostras de fezes diretamente do reto de cada animal. Nestas amostras fecais, foram determinadas as contagens de ovos por grama de fezes (OPG) de acordo com técnica descrita por Lima (1989). Paralelamente ao exame de OPG, foram realizadas as coproculturas segundo técnica de Guimarães (1971) e identificação das larvas de acordo com Keith (1953).

De catorze em catorze dias, em cada piquete dos diversos grupos de animais, foram coletadas amostras de pastagem, em ziguezague, de pontos variados. De cada amostra, foram utilizados em torno de $500 \mathrm{~g}$ de pastagem, de onde se recuperaram, quantificaram e posteriormente identificaram as larvas de nematóides parasitos de bovinos segundo Lima (1989) e Keith (1953). A matéria seca foi obtida e os dados foram transformados em número de larvas por kg de matéria seca segundo Araújo (1996).

De quinze em quinze dias, um animal traçador (bezerro mestiço holandês $x$ zebu, de seis meses de idade, macho) foi introduzido nos lotes de cada grupo, juntamente com a população permanente de animais, para medir a infestação das pastagens por nematóides parasitos gastrintestinais de bovinos. Estes animais, antes de serem introduzidos nas pastagens, foram confinados e receberam dois tratamentos anti-helmínticos com albendazole $(7,5 \mathrm{mg} / \mathrm{kg}$ de peso vivo, por via oral), intervalados de catorze dias, e foram utilizados sete dias após o último tratamento. Cada animal permaneceu por catorze dias nas pastagens e depois foi retirado, estabulado por catorze dias e sacrificado. Os estádios imaturos e os vermes adultos foram recuperados, quantificados e identificados segundo Araújo (1996).

Foram registrados, diariamente, os dados meteorológicos, colhidos em estação especializada na própria fazenda experimental, referentes às médias mensais das temperaturas máximas, médias e mínimas, à umidade relativa do ar e à precipitação pluvial.

Foram comparados os dados de OPG, das coproculturas, número de larvas recuperadas da pastagem e dos animais traçadores dos dois grupos. Os resultados foram interpretados estatisticamente por meio de análise de variância (teste F) a 1 e $5 \%$ de probabilidade e regressão linear. As médias dos fatores qualitativos foram comparadas pelo teste de Tukey em nível de $5 \%$ de probabilidade.

\section{Resultados e discussão}

Os valores médios mensais das contagens de OPG dos animais permanentes estão representados na Figura 1. No mês de maio, o OPG dos animais do grupo controle foi $73,68 \%$ maior em relação aos animais do grupo tratado com A. robusta $(\mathrm{P}<0,05)$. 


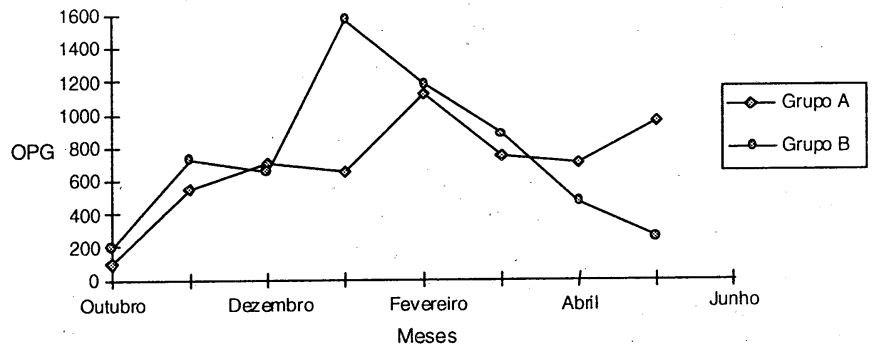

Figura 1: Valores médios mensais das contagens de ovos por grama de fezes (OPG) dos nematóides da superfamília Strongyloidea nos animais do grupo controle (A) e de bezerros tratados com conídios (B).

As curvas de regressão (Figuras 2 e 3 ) demonstram que houve aumento no OPG dos animais nos dois grupos, nos meses de outubro a janeiro. Nos animais do grupo A houve estabilização da curva de OPG nos meses subseqüentes e ligeiro aumento no mês de maio, e no grupo $B$ a contagem foi reduzida a valores significativos $(P<0,05)$.

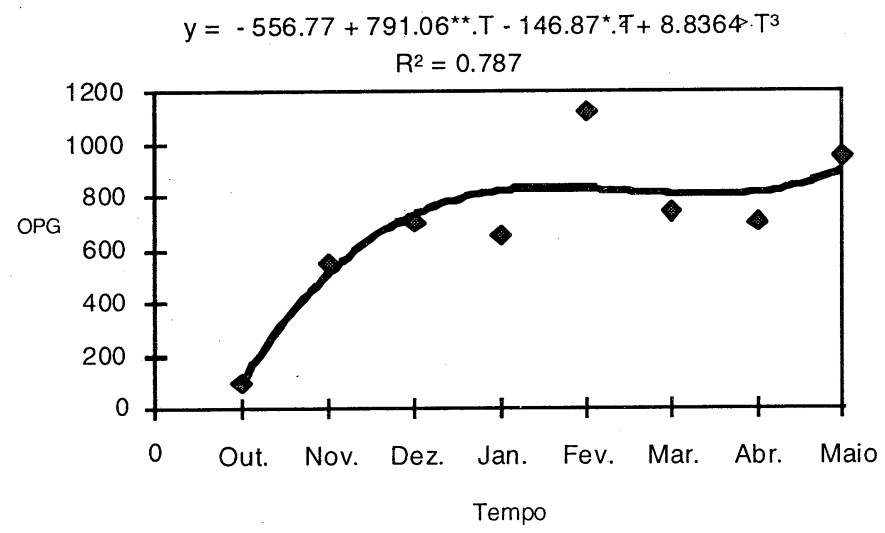

Figura 2: Curva de regressão da estimativa do número de ovos por grama de fezes (OPG), de outubro a maio, para os animais do grupo controle (A).

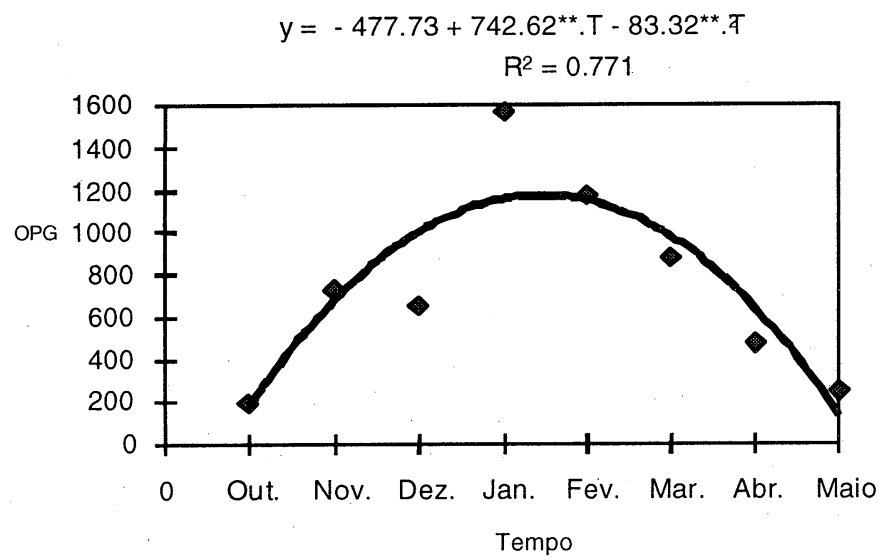

Figura 3: Curva de regressão da estimativa do número de ovos por grama de fezes (OPG), de outubro a maio, para os animais do grupo tratado com conídios de $A$. robusta (B).
Através dos valores médios mensais do OPG, obtiveram-se as participações percentuais das $L 3$, após coprocultura dos animais permanentes dos grupos $\mathrm{A}$ e $\mathrm{B}$, que estão representados nas Figuras 4 e 5 , respectivamente. A média das porcentagens de larvas recuperadas após coprocultura ao final do experimento indica a prevalência do gênero Haemonchus com $48,78 \%$, seguido dos gêneros Cooperia com $36,36 \%$, e Oesophagostomum com $14,86 \%$. As larvas do gênero Trichostrongylus foram encontradas somente em animais do grupo controle, em baixos níveis e no mês de abril.

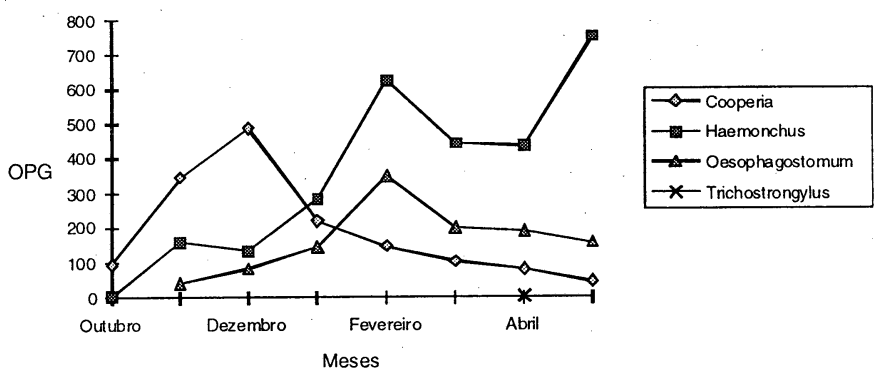

Figura 4: Valores médios mensais das contagens de ovos de nematóides recuperados da coprocultura dos animais do grupo controle (grupo A).

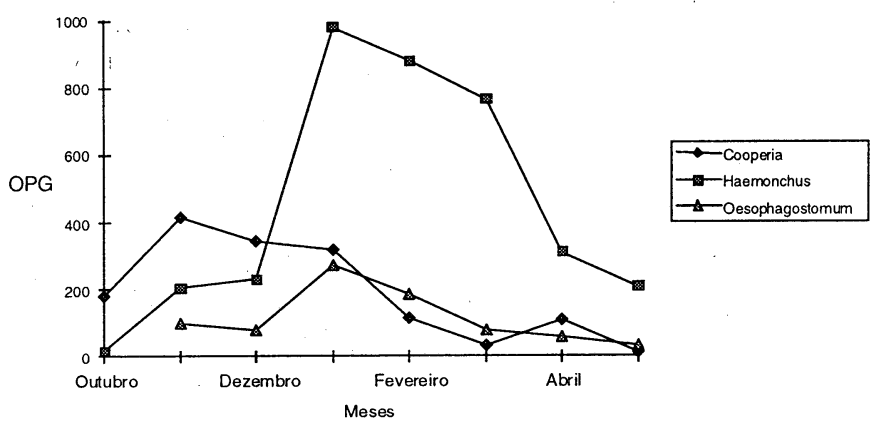

Figura 5: Valores médios mensais das contagens de ovos de nematóides recuperados da coprocultura dos animais do grupo tratado com conídios de Arthrobotrys robusta (grupo B).

O aumento no número de Haemonchus pode ser explicado pela infecção inicial dos bezerros permanentes com H. placei e pela elevação da precipitação pluvial para valores desejáveis ao desenvolvimento das larvas na pastagem segundo Roberts et al. (1952), como se observa na Figura 6.

A predominância de larvas de Oesophagostomum nas coproculturas dos bezerros permanentes sobre o gênero Cooperia, a partir de fevereiro, sugere que este gênero foi menos predado pelo fungo em relação ao gênero Cooperia, talvez por apresentar menor motilidade e ser indutor de menor número de armadilhas, de acordo com Araújo et al. (1998) e Nansen et al. (1988). Todavia, posteriores testes específicos in vitro e in vivo com Oesophagostomum são necessários para confirmar esse resultado.

Ao final do experimento, os nematóides do gênero Cooperia foram os mais prevalentes nos animais traçadores, com $56,15 \%$, seguindo-se os gêneros Haemonchus com $40,84 \%$, Oesophagostomum com 2,31\%, e Trichuris com $0,57 \%$. O número de helmintos recuperados dos animais traçadores foi 
maior no período de outubro a março, demonstrando uma estreita relação de larvas recuperadas com a precipitação pluvial. Não houve diferença estatisticamente significativa $(P>0,05)$ nas médias de nematóides encontrados entre os dois grupos de animais traçadores.

Os valores das contagens de $L_{3}$ por kg de matéria seca de pastagem estão representados na Tabela 1. O número de larvas totais recuperadas no pasto dos animais do grupo tratado com conídios foi menor $77,39 \%$ em relação ao grupo controle, tendo o gênero Cooperia se apresentado como o mais prevalente em ambas as pastagens. Houve redução de $73,28 \%$ no número total de larvas do gênero Cooperia no grupo tratado com conídios, em relação ao grupo controle $(P<0,05)$. Não houve diferença estatisticamente significativa $(P>0,05)$ nas médias de larvas infectantes dos gêneros Haemonchus e Oesophagostomum recuperadas por $\mathrm{kg}$ de matéria seca de pasto entre os grupos A e B.

Tabela 1: Número de larvas infectantes recuperadas dos pastos com animais do grupo controle e animais do grupo tratado com conídios de A. robusta, de outubro de 1996 a maio de 1997.

\begin{tabular}{|c|c|c|c|c|c|c|}
\hline \multirow[b]{2}{*}{ Meses } & \multicolumn{3}{|c|}{ Controle } & \multicolumn{3}{|c|}{ Tratado } \\
\hline & Cooperia & Haemonchus & Oesophagostomum & Cooperia & Haemonchus & Oesophagostomum \\
\hline Outubro & 271 & 41 & 82 & 187 & - & 125 \\
\hline Novembro & 30 & - & - & - & - & - \\
\hline Dezembro & - & 17 & - & - & - & - \\
\hline Janeiro & 28 & - & - & 20 & - & - \\
\hline Fevereiro & 322 & 33 & - & 12 & - & - \\
\hline Março & 86 & 18 & 99 & - & - & - \\
\hline Abril & 130 & 33 & 30 & 22 & 11 & 11 \\
\hline Maio & 35 & 41 & 212 & - & 26 & 52 \\
\hline Total & 902 & 183 & 423 & 241 & 37 & 63 \\
\hline
\end{tabular}

A Figura 6 demonstra que não ocorreram variações no número de larvas nas pastagens em função da pluviosidade. Podemos deduzir que, no caso de Cooperia, a redução do número de larvas recuperadas no pasto do grupo $\mathrm{B}$ foi devido ao tratamento $(P<0,05)$.

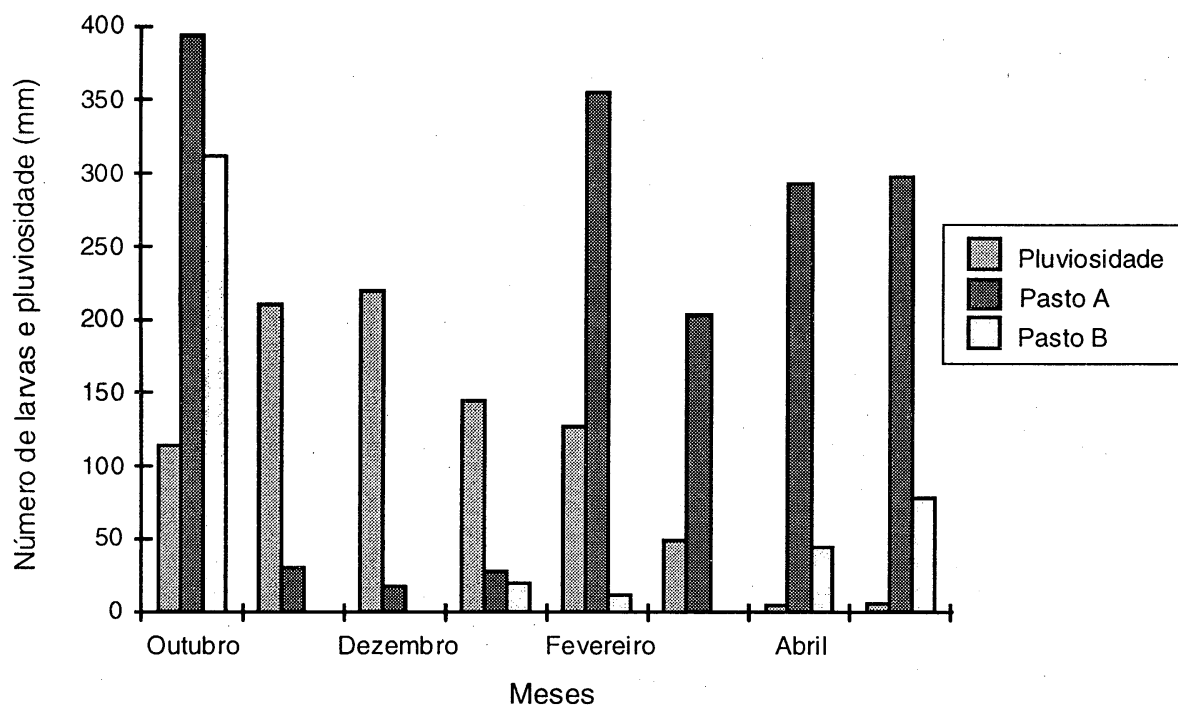

Figura 6: Número de larvas recuperadas dos pastos dos animais do grupo controle (pasto A) e tratado com conídios de $A$. robusta (pasto $B$ ), durante os oito meses de experimento, em relação à pluviosidade mensal.
Durante o período experimental, as temperaturas médias mensais oscilaram entre $20,04^{\circ} \mathrm{C}$ (outubro de 1996) e $24,63^{\circ} \mathrm{C}$ (fevereiro de 1997 ) e foram propícias para o desenvolvimento dos estádios de vida livre dos nematóides parasitos gastrintestinais de bovinos. Entretanto, estas temperaturas não foram favoráveis ao desenvolvimento dos estádios de vida livre de Trichostrongylus, pois as temperaturas ideais para a evolução desse parasito estão compreendidas entre $6^{\circ}$ e $20^{\circ} \mathrm{C}$ (Levine, 1963) e $10^{\circ}$ e $18,8^{\circ} \mathrm{C}$ (Fernandes, 1975).

A temperatura ótima, em geral, de desenvolvimento de Arthrobotrys, oscila entre $20^{\circ} \mathrm{C}$ e $29^{\circ} \mathrm{C}$ (Araújo, 1996). Portanto, a temperatura foi favorável ao desenvolvimento de larvas infectantes na pastagem e no crescimento dos fungos.

Durante o período experimental, os meses de março, abril e maio não atingiram o limite mínimo de $50 \mathrm{~mm}$ de precipitação pluvial mensal requerido para o desenvolvimento de ovos e larvas dos helmintos de bovinos (Gordon, 1948 e Williams e Mayhew, 1967). Os meses de novembro, dezembro, janeiro e fevereiro apresentaram os maiores valores de precipitação pluvial e coincidiram com os picos de OPG e aumento no número de larvas de Haemonchus na coprocultura dos animais permanentes, e com o aumento nos nematóides recuperados dos animais traçadores de ambos os grupos.

Em prévios trabalhos desenvolvidos por Araújo (1996), Araújo et al. (1996), Araújo et al. (1998) e Araújo et al. (1999) este isolado de $A$. robusta foi promissor quanto à passagem pelo trato gastrintestinal de bovinos e reduziu o OPG em 53,81\% em animais tratados em relação aos animais do grupo controle e uma redução de $70,45 \%$ no número de vermes recuperados da necrópsia de animais traçadores.

\section{Conclusões}

- Os fungos nematófagos devem ser empregados logo no início do período chuvoso, quando as condições das pastagens são adequadas para o pastoreio dos animais e favoráveis ao desenvolvimento de ovos e larvas de helmintos no meio ambiente.

- O número de animais traçadores utilizados no ensaio experimental foi insufi- 
ciente, determinando coeficientes de variação com altos valores, e impediu que os resultados da análise estatística demonstrassem relação entre o grupo controle e o grupo tratado com conídios do fungo.

- A temperatura, a precipitação pluviométrica e a umidade relativa do ar não foram fatores limitantes ao desenvolvimento e à sobrevivência de larvas infectantes nas pastagens e para o crescimento do isolado $A$. robusta. Observou-se a eficácia do isolado utilizado devido à redução significativa $(\mathrm{P}<0,05)$ no número total de larvas recuperadas nos animais do pasto tratado.
- A interação entre nematóides, fungo nematófago, animais e fatores ambientais proporciona resultados que, quando analisados em termos da combinação simples entre nematóides e fungo nematófago, podem ter difícil interpretação. $O$ animal e o ambiente em cada situação de estudo representam um papel muitas vezes decisivo na discussão dos resultados obtidos.

- A. robusta parece ser um fungo promissor para ser utilizado no controle das verminoses gastrintestinais de bovinos. No entanto, mais estudos com outros isolados e outras espécies de nematóides são necessários.

\section{Agradecimentos}

À Doutora Maria Cecília Reale Vieira-Bressan, por ceder as larvas infectantes de Haemonchus placei. À Fundação de Amparo à Pesquisa de Minas Gerais e à Fundação Banco do Brasil pelo financiamento deste projeto.

\section{Referências bibliográficas}

ARAÚJO, J.V. Interação entre larvas infectantes de Cooperia punctata e fungos predadores do gênero Arthrobotrys, caracterização de isolados de Arthrobotrys e seu uso no controle biológico de nematódeos parasitos gastrintestinais de bovinos. 1996. $110 \mathrm{f}$. Tese (Doutorado) - Instituto de Ciências Biológicas, Universidade Federal de Minas Gerais.

, NETO, A.P., AZEVEDO, M.H.F. Screening parasitic nematodetrapping fungi Arthrobotrys for passage through the gastrointestinal tract of calves. Arq. Bras. Med. Vet. Zoot.v. 48, n. 6, p. 543-552, 1996.

, GOMES, A.P.S., GUIMARÃES, M.P. Biological control of bovine gastrointestinal nematode parasites in southeastern Brazil by the nematode-trapping fungus Arthrobotrys robusta. Rev. Bras. Parasitol. Vet., v. 7, n. 2, p. 117-122, 1998.

STEPHANO, M.A., SAMPAIO, W.M. Passage of nematodetrapping fungi through the gastrointestinal tract of calves. Vet. Arhiv., v. 69, n. 2, p. 69-78, 1999.

BARRON, G.L. (ed.) The nematode-destroying fungi. Canadian Biological Publications. Guelph, 1977.

DUDDINGTON, C.L. Notes on the technique of handling predaceous fungi. Trans. Brit. Mycol. Soc., v. 38, n. 2, p. 97-103, 1955.

FERNANDES, F.G. Tempo e clima na Trichostrongilose ovina. 1975. $87 \mathrm{f}$. Tese (Livre-Docência) - Faculdade de Veterinária - Universidade Federal de Pelotas.

GORDON, H.M. The epidemiology of parasitic diseases, with special reference to studies with nematode parasites of sheep. Aust. Vet. J., v. 29, n. 6, p. 337-348, 1948.

GRAY, N.F. Nematophagous fungi with particular reference to their ecology. Biol. Rev., v. 62, n.7, p. 245-304, 1987.
GUIMARÃES, M.P. Variação estacional de larvas infectantes de nematóides parasitos de bovinos em pastagens de cerrado de Sete Lagoas, Minas Gerais. 1971. $45 \mathrm{f}$. Tese (Mestrado) - Instituto de Ciências Biológicas, Universidade Federal Minas de Gerais.

KEITH, R.K. The differentiation on the infective larvae of some common nematode parasites of cattle. Aust. J. Zool., v. 1, n. 4, p. 223-235, 1953.

LEVINE, N.D. Weather, climate and the bionomics of ruminant nematode larvae. Adv. Vet. Sc., v. 8, n. 3, p. 215-261, 1963.

LIMA, W.S. Dinâmica das populações de nematódeos parasitos gastrintestinais em bovinos de corte, alguns aspectos da relação parasito-hospedeiro e do comportamento dos estádios de vida livre na região do vale do Rio Doce, MG, Brasil. 1989. 178 f. Tese (Doutorado) - Instituto de Ciências Biológicas, Universidade Federal de Minas Gerais.

NANSEN, P., GRONVOLD, J., HENRIKSEN, S.A., WOLSTRUP, J. Interactions between the predacious fungus Arthrobotrys oligospora and third-stage larvae of a series of animal-parasitic nematodes. Vet. Parasitol., v. 26, n. 7, p. 329-337, 1988.

ROBERTS, F.H.S., O' SULLIVAN, P.J., RIEK, R.F. The epidemiology of parasitic gastro-enteritis of cattle. Aust. J. Agricul. Res., v. 3, n. 3, p. 187-226, 1952.

SANTOS, M.A., FERRAZ, S., MUCHOVEJ, J. Detection and Ecology of nematophagous fungi from Brazilian soils. Nematol. Bras., v. 15, $\mathrm{n}$. 2, p. 121-134, 1991.

WILLIAMS, J.C., MAYHEW, R.L. Survival of infective larvae of the cattle nematodes, Cooperia punctata, Trichostrongylus axei and Oesophagostomum radiatum. Am. J. Vet. Res., v. 28, n. 11, p. 629640, 1967. 